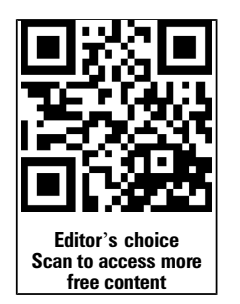

- Additional material is published online only. To view please visit the journal online (http://dx.doi.org/10.1136/ neurintsurg-2014-011179).

${ }^{1}$ Department of Neurological Surgery, Rush University Medical Center, Chicago, Illinois, USA

${ }^{2}$ Department of Biomedical Engineering, University of Wisconsin-Madison, Madison, Wisconsin, USA

${ }^{3}$ Department of Radiology, University of WisconsinMadison, Madison, Wisconsin, USA

${ }^{4}$ Department of Engineering Physics, University of Wisconsin-Madison, Madison, Wisconsin, USA

\section{Correspondence to} Dr R Moftakhar, Department of Neurological Surgery, Rush University Medical Center, Chicago, IL 60612, USA; roham.moftakhar@gmail.com

Received 16 February 2014 Revised 14 May 2014 Accepted 16 May 2014 Published Online First 2 June 2014

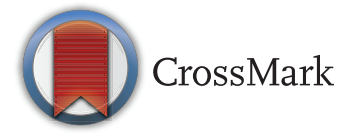

To cite: Moftakhar $R, X_{\text {, }}$, Aagaard-Kienitz B, et al. J Neurolntervent Surg

2015;7:584-590.

\title{
Preliminary in vivo evaluation of a novel intrasaccular cerebral aneurysm occlusion device
}

\author{
Roham Moftakhar, ${ }^{1}$ Fangmin $\mathrm{Xu}_{1}{ }^{2}$ Beverly Aagaard-Kienitz, ${ }^{3}$ Daniel W Consigny, ${ }^{3}$ \\ Julie R Grinde, ${ }^{3}$ Kevin Hart, ${ }^{4}$ Claire E Flanagan, ${ }^{2}$ Wendy C Crone, ${ }^{2,4}$ \\ Kristyn S Masters ${ }^{2}$
}

\begin{abstract}
Objective Current endovascular technology does not offer a perfect solution for all cerebral aneurysms. Our group has built two versions of a novel aneurysm intrasaccular occlusion device (AIOD) to address the drawbacks associated with current occlusion devices. The objective of the present study was to perform pilot proof of concept in vivo testing of this new AIOD in swine and canines.
\end{abstract}

Methods Two configurations of the AIOD, termed 'coil-in-shell' and 'gel-in-shell', were implanted in surgically created sidewall aneurysms $(n=4)$ in swine for acute occlusion studies, as well as sidewall $(n=8)$ and bifurcation aneurysms $(n=3)$ in canines to assess long term occlusion efficacy. Occlusion at all time points (immediate, 6 weeks, and 12 weeks) was evaluated by angiography. Neointimal healing at 12 weeks postimplantation in canines was examined histologically. Results Angiographic analysis showed that both the coil-in-shell and gel-in-shell devices achieved complete aneurysm occlusion immediately following device delivery in sidewall aneurysms in swine. In longer term canine studies, initial occlusion ranged from $71.3 \%$ to $100 \%$, which was stable with no recurrence in any of the sidewall aneurysms at 6 or 12 weeks. Histological analysis at 12 weeks showed mature fibromuscular tissue at the neck of all aneurysms and no significant inflammatory response.

Conclusions The AIOD tested in this study showed promise in terms of acute and chronic occlusion of aneurysms. Our findings suggest that these devices have the potential to promote robust tissue healing at the aneurysm neck, which may minimize aneurysm recurrence. Although proof of principle has been shown, further work is needed to deliver this device through an endovascular route.

\section{INTRODUCTION}

Although significant advances have been made in the endovascular treatment of intracranial aneurysms, incomplete aneurysm occlusion and recanalization remain potential shortcomings of many currently used occlusion devices. Endovascular devices currently used for the treatment of intracranial aneurysms include coils, liquid embolic agents, and flow diverting devices. One of the drawbacks of coil embolization is coil compaction over time, leading to recanalization of the aneurysm. Some degree of aneurysm recanalization occurs in as many as $20 \%$ of cases. ${ }^{1-3}$ In larger aneurysms, placement of multiple coils can be time consuming, and longer procedural times may lead to increased morbidity and mortality. ${ }^{3}$

An alternative to coil embolization is the use of liquid embolic agents. It is thought that filling aneurysms with such polymers will reduce many of the shortcomings associated with coiling, such as coil compaction. ${ }^{4}$ Use of the liquid embolic agent Onyx 500 (Covidien, Irvine, California, USA) involves controlled injection with use of a balloon, which has to be inflated and deflated periodically. Occlusion of aneurysms with liquid embolic agents has also faced other problems, such as difficulty containing the liquid material, thus leading to leakage into the normal circulation prior to solidification and causing incomplete filling of the aneurysm and thromboembolic complications. ${ }^{5-9}$

A recent development in aneurysm occlusion technology is the intrasaccular flow diversion device, examples of which include WEB (Woven EndoBridge, Sequent Medical Inc, Aliso Viejo, California, USA) ${ }^{10} 11$ and Luna AES (NFocus Neuromedical, Palo Alto, California, USA). ${ }^{12} 13$ These self-expanding metal mesh devices are thought to work by modifying flow at the level of the aneurysm neck and are placed within the aneurysm sac. Complete or near complete aneurysm occlusion can be achieved with these devices. One advantage of intrasaccular devices over stents or endoluminal flow diverters is that dual antiplatelet agents are not needed.

Our group has developed two versions of an aneurysm intrasaccular occlusion device (AIOD). In the coil based AIOD design, a flexible polymer shell is expanded via a single shape memory nickel titanium (NiTi) wire within the aneurysm ${ }^{14}$ (see online supplementary figure S1). Creation of this design was motivated by the hypothesis that delivery of a single coil would decrease procedure time, and the presence of an elastic shell would decrease device compaction. Meanwhile, the gel based device involves expansion of this polymer shell via injection of in situ gelling sodium alginate into the shell. The design of the gel-in-shell device was motivated by the containment challenge currently associated with the delivery of liquid embolic agents, as well as the biocompatibility of current liquid embolic compounds. These two AIOD designs are completely intra-aneurysmal. Furthermore, both AIOD devices possess a unique shell composed of a copolymer of hyaluronic acid and polyurethane; this material has been shown to be bioactive ${ }^{15-17}$ and could accelerate neointimal 
formation to eliminate the aneurysm from the circulation. This study describes the in vivo testing of these two AIODs for aneurysm treatment.

\section{MATERIALS AND METHODS \\ Coil-in-shell device}

An AIOD consisting of a single NiTi wire frame surrounded by a polymeric shell was constructed as described previously. ${ }^{14}$ Briefly, 0.0100 inch diameter ( $\mathrm{Ni} 55.8 \mathrm{wt} \%$, O $0.05 \mathrm{wt} \%$ maximum, C $0.02 \mathrm{wt} \%$ maximum, Ti balance; SE508 Straight Bright, Nitinol Devices and Components, Fremont, California, USA) NiTi coils were wrapped around a helical jig and heat treated in a tube furnace at $550^{\circ} \mathrm{C}$ in nitrogen for $35 \mathrm{~min}$, followed by cooling in air for $30 \mathrm{~min}$. These NiTi coils $(6-10 \mathrm{~mm}$ in diameter) were then placed within matching hemispherical films composed of a polyurethane-hyaluronic acid (PU-HA) copolymer, and the seams of the films were joined via application of $1 \mathrm{~g} / \mathrm{mL}$ PU in dimethyl formamide. A previous publication describes the synthesis and characterization of PU-HA, demonstrating that it is highly elastic, transparent, biocompatible, and hemocompatible. ${ }^{17}$ All device components, as well as the final device, were soaked in $70 \%$ ethanol for $30 \mathrm{~min}$ followed by air drying in a biosafety cabinet. Construction of the device was also conducted in a biosafety cabinet using a sterile technique, and the devices were individually sealed in sterile containers prior to use. Devices were made in a range of sizes (6-10 mm diameter) to accommodate variations naturally found in the diameter of the vessels used during surgical aneurysm creation.

\section{Gel-in-shell device}

Devices consisting of an in situ gelling hydrogel encapsulated within a polymer shell were constructed using sodium alginate and PU-HA. PU-HA films were formed into spheres as described above. Immediately prior to device delivery, $2 \mathrm{~mL}$ of a $2 \mathrm{wt} \%$ solution of sodium alginate (Sigma-Aldrich, St Louis, Missouri, USA) in $\mathrm{diH}_{2} \mathrm{O}$ was mixed with $0.92 \mathrm{~mL}$ of $0.5 \% \mathrm{CaCO}_{3}$ (Sigma-Aldrich) in $\mathrm{diH}_{2} \mathrm{O}$. This mixture was combined with $0.02 \mathrm{~mL}$ of $4.3 \% \mathrm{D}$-glucono- $\gamma$-lactone (Sigma-Aldrich). Using a $26 \mathrm{~g}$ needle, the final alginate mixture was injected into the PU-HA shell that was seated in the aneurysmal pouch, as described below. This alginate formulation was selected to achieve a slow controlled gelation that was complete within $15 \min ^{18}$

\section{In vivo aneurysm creation}

All animal experiments were conducted in accordance with policies set by the Animal Care and Use Committee at the University of Wisconsin-Madison. In two swine and seven adult mongrel canines, sidewall and bifurcation aneurysms were created surgically by anastomosing vein pouches to the common carotid arteries, as previously described. ${ }^{19} 20$ The porcine and canine animal models were used to study acute and long term occlusion efficacy, respectively. ${ }^{20}$ The aneurysms were made from venous tissue, which was sewn to the arterial wall. The top of the venous pouch was left open for the devices to be inserted from the top of the aneurysm pouch. The pouches were closed using one silk suture. The devices were inserted into the pouch while the parent vessel was clamped on each side of the aneurysm. Carotid blood flow was restored, and angiography was performed immediately post-implantation to assess aneurysm occlusion. Because of this delivery approach, angiography before device insertion could not be performed. However, based on previous publications from our laboratory, the average aneurysm dimensions for sidewall aneurysms were $6.9 \mathrm{~mm}$ in height, $6.4 \mathrm{~mm}$ in diameter, and $4.7 \mathrm{~mm}$ neck width. The average dimensions for bifurcation aneurysms were $10.3 \mathrm{~mm}$ in height, $7.7 \mathrm{~mm}$ in diameter, and $5.5 \mathrm{~mm}$ neck. ${ }^{20}$ Two sidewall aneurysms were created in each of four canines. One bifurcation aneurysm was created in each of three canines.

\section{Angiographic and histological analysis}

The two swine were euthanized immediately following postimplantation angiography. Angiographic analysis of aneurysm occlusion in the seven canines was performed immediately postimplantation, and at 6 and 12 weeks post-implantation, with all canines euthanized at 12 weeks. Angiographic images were analyzed using ImageJ software (NIH, Bethesda, Maryland, USA) to quantify the per cent of each aneurysm that was filled with the device at each time point; these data are reported as per cent occlusion. In addition, angiography was also categorized by two of the authors (RM and BA-K) using the Raymond Scale for aneurysm obliteration. ${ }^{21}$ The Raymond grading was consistent between the two evaluators in each case.

At sacrifice, carotid arteries and aneurysms were resected en bloc, placed in $10 \%$ formalin, and sent to Wasatch Histo Consultants Inc (Winnemucca, Nevada, USA) for histological processing. The tissues were embedded in methyl methacrylate and sectioned transversely using a diamond wire saw to yield approximately 4-6 wafers per sample. The wafers were glued to a plastic slide, ground to approximately $50 \mu \mathrm{m}$ using an Exakt grinder, polished to an optical finish, and stained with hematoxylin and eosin. One sample was kept intact for gross examination of vessel patency.

Histological slides were assessed by an independent clinical pathologist. The slices were treated with metachromatic stain to further enhance visualization of tissue structures. The thickness of the neointimal tissue covering the occlusion device at the neck of each aneurysm was measured by the same pathologist in three places.

\section{RESULTS}

\section{Angiography}

Although these coil-in-shell and gel-in-shell AIODs are ultimately intended to be delivered via an endovascular route, the purpose of this proof of concept study was solely to determine whether the fully deployed devices could occlude aneurysms surgically created in an animal model. In initial animals, the most common problem encountered at the time of implantation was slight undersizing of the device relative to the aneurysm. In subsequent animals, this issue was resolved by having multiple device sizes available at the time of surgery.

In one swine, two coil-in-shell devices were placed in opposing configurations: one with the coil axis positioned perpendicular to the parent vessel and one with the coil axis oriented parallel to the parent vessel. Both devices completely occluded the aneurysm immediately post-implantation (see online supplementary figure S2A), confirming that variations in device orientation, which may occur unintentionally during device deployment, did not negatively impact on occlusion efficacy. In the second swine, two sidewall aneurysms were also completely occluded by gel-in-shell devices (see online supplement figure S2B).

For long term studies, a total of 11 devices were implanted in seven canines, with four canines each receiving two devices deployed in sidewall aneurysms and three canines each receiving one device deployed in a bifurcation aneurysm. Of the eight devices implanted in sidewall aneurysms, five were gel-in-shell and three were coil-in-shell. With respect to occlusion 
immediately post-implantation, the devices were generally successful in blocking blood flow into the aneurysms, with obliteration rates ranging from $71.3 \%$ to $100 \%$ (table 1 ). Of the three bifurcation aneurysms, two had filling of the sac of the aneurysm at initial implantation (Raymond grade 3) and one was completely occluded (Raymond grade 1). Of the sidewall aneurysms, four had filling of the sac at the time of implantation (Raymond grade 3), three had residual neck (Raymond grade 2 ), and one was completely occluded (Raymond grade 1). Dissection of blood flow around the occlusion device was observed in one aneurysm (dog No 5, LCC). Undersizing of the device relative to the aneurysm was believed to be the primary cause of incomplete occlusion; as noted earlier, this issue was addressed by having multiple device sizes during subsequent surgeries, whereupon occlusion efficacy was notably improved.

All sidewall aneurysms demonstrated a stable degree of occlusion at 6 and 12 weeks post-implantation. In several instances there was improvement in aneurysm occlusion (table 1, figure 1). The Raymond grade either stayed the same or improved in all sidewall aneurysms at 6 and 12 weeks. Of those sidewall aneurysms that had neck remnant on day 0 (Raymond grade 2), all remained stable or went on to completely occlude (Raymond grade 1), for a total of four sidewall aneurysms completely occluded by week 12 . One additional sidewall aneurysm with initial sac filling (Raymond grade 3 ) also improved to Raymond grade 2 by the 6 and 12 week time points. No obvious differences were observed between the performance of the coil-in-shell and gel-in-shell devices at any time point.

Occlusion of bifurcation aneurysms proved more challenging with respect to both device design and placement. The shape of the first generation of these devices was spherical, which was found to be an imperfect match for the ellipsoid shape of the bifurcation aneurysm. Thus the two subsequent implantations in bifurcation aneurysms used a conical shaped shell that better matched the geometry of the aneurysm. An initial occlusion of $100 \%$ was achieved with one of the two conical shaped devices (table 1, dog No 6), but the device suffered from poor initial placement and was found to protrude slightly into the parent vessel. As the device became better seated within the aneurysm, per cent occlusion at week 6 decreased slightly to $94.5 \%$, but remained stable at week 12 , with a Raymond grade of 1 . In dog No 1, angiography at week 12 was not performed due to the angiography equipment malfunctioning. In dog No 7, angiography at weeks 6 and 12 was not performed due to device migration sometime after the immediate angiography. The aneurysm created in $\operatorname{dog}$ No 7 was noted to have a weak wall and dusky appearance at the time of surgery, potentially leading to device migration. In some post-implantation angiograms there was parent artery stenosis seen adjacent to the device, which may be alleviated by more precise sizing and placement of the device.

\section{Histology}

Eight of 10 aneurysm samples submitted for histological examination showed fibromuscular neointimal tissue covering the occlusive device at the neck of the aneurysm at 12 weeks postimplantation (figure 2 and see online supplementary figure S3). The remaining two samples could not be evaluated because the plane of the histological sectioning may not have been through the neck. In those sections amenable to analysis, the tissue covering the neck of the aneurysm was composed of mature fibromuscular tissue with no significant inflammatory cells, and the thickness of this covering ranged from $0.33 \pm 0.2$ to 1.42 $\pm 0.25 \mathrm{~mm}$. The average tissue thickness for each type of device is displayed in table 2. Moreover, neointimal coverage was equally robust for both incompletely and completely occluded aneurysms, as seen in figure 2 . In many cases, the luminal surface of the tissue, facing the parent vessel, was lined with endothelial cells (see online supplementary figure S4). The parent vessel appeared patent in all cases.

\section{DISCUSSION}

AIODs address several limitations associated with existing technology, such as coils, liquid embolic agents, and flow diverting stents. This study examined the proof of concept in vivo performance of a new AIOD which we designed and tested in two different configurations, described herein as 'coil-in-shell' and 'gel-in-shell' devices. At 12 weeks post-implantation of either the coil-in-shell or gel-in-shell devices, no recanalization was observed in sidewall aneurysms, with several aneurysms

Table 1 Per cent occlusion and Raymond scores for aneurysms treated with coil-in-shell or gel-in-shell devices

\begin{tabular}{|c|c|c|c|c|c|c|c|}
\hline \multirow[b]{2}{*}{ Animal } & \multirow[b]{2}{*}{ Condition } & \multicolumn{3}{|c|}{ Per cent occluded } & \multicolumn{3}{|c|}{ Raymond score } \\
\hline & & Postop & 6 weeks & 12 weeks & Postop & 6 weeks & 12 weeks \\
\hline Swine 1 (LCC) & Coil-in-shell, sidewall & 100 & NA & NA & 1 & NA & NA \\
\hline Swine 1 (RCC) & Coil-in-shell, sidewall & 100 & NA & NA & 1 & NA & NA \\
\hline Swine 2 (LCC) & Gel-in-shell, sidewall & 100 & NA & NA & 1 & NA & NA \\
\hline Swine 2 (RCC) & Gel-in-shell, sidewall & 100 & NA & NA & 1 & NA & NA \\
\hline Dog No 1 & Coil-in-shell, bifurcation & $73.4( \pm 5.7)$ & $71.8( \pm 1.9)$ & NA & 3 & 3 & NA \\
\hline Dog No 2 (LCC) & Coil-in-shell, sidewall & $80.9( \pm 4.8)$ & $93.8^{*}( \pm 2.5)$ & $92.4( \pm 2.5)$ & 3 & 2 & 2 \\
\hline Dog No 2 (RCC) & Coil-in-shell, sidewall & $82.6( \pm 1.6)$ & $84.4( \pm 3.5)$ & $82.4( \pm 1.4)$ & 3 & 3 & 3 \\
\hline Dog No 3 (LCC) & Gel-in-shell, sidewall & $92.2( \pm 1.2)$ & $90.3( \pm 1.5)$ & $89.2( \pm 3.4)$ & 3 & 3 & 3 \\
\hline Dog No 3 (RCC) & Gel-in-shell, sidewall & $98.7( \pm 0.3)$ & $100^{*}( \pm 0.0)$ & $100( \pm 0.0)$ & 2 & 1 & 1 \\
\hline Dog No 4 (LCC) & Gel-in-shell, sidewall & $97.9( \pm 0.8)$ & $98.8( \pm 0.6)$ & $98.6( \pm 0.2)$ & 2 & 1 & 1 \\
\hline Dog No 4 (RCC) & Coil-in-shell, sidewall & $99( \pm 0.4)$ & $98.8( \pm 0.0)$ & $99.4( \pm 0.9)$ & 2 & 2 & 1 \\
\hline Dog No 5 (LCC) & Gel-in-shell, sidewall & $71.3( \pm 1.0)$ & $73.2( \pm 4.3)$ & $69.4( \pm 1.1)$ & 3 & 3 & 3 \\
\hline Dog No 5 (RCC) & Gel-in-shell, sidewall & $100( \pm 0.0)$ & $100( \pm 0.0)$ & $100( \pm 0.0)$ & 1 & 1 & 1 \\
\hline Dog No 6 & Gel-in-shell, bifurcation & $100( \pm 0.0)$ & $94.5^{*}( \pm 1.3)$ & $92.8( \pm 2.7)$ & 1 & 3 & 1 \\
\hline Dog No 7 & Gel-in-shell, bifurcation & $73.4( \pm 2.2)$ & NA & NA & 3 & NA & NA \\
\hline
\end{tabular}



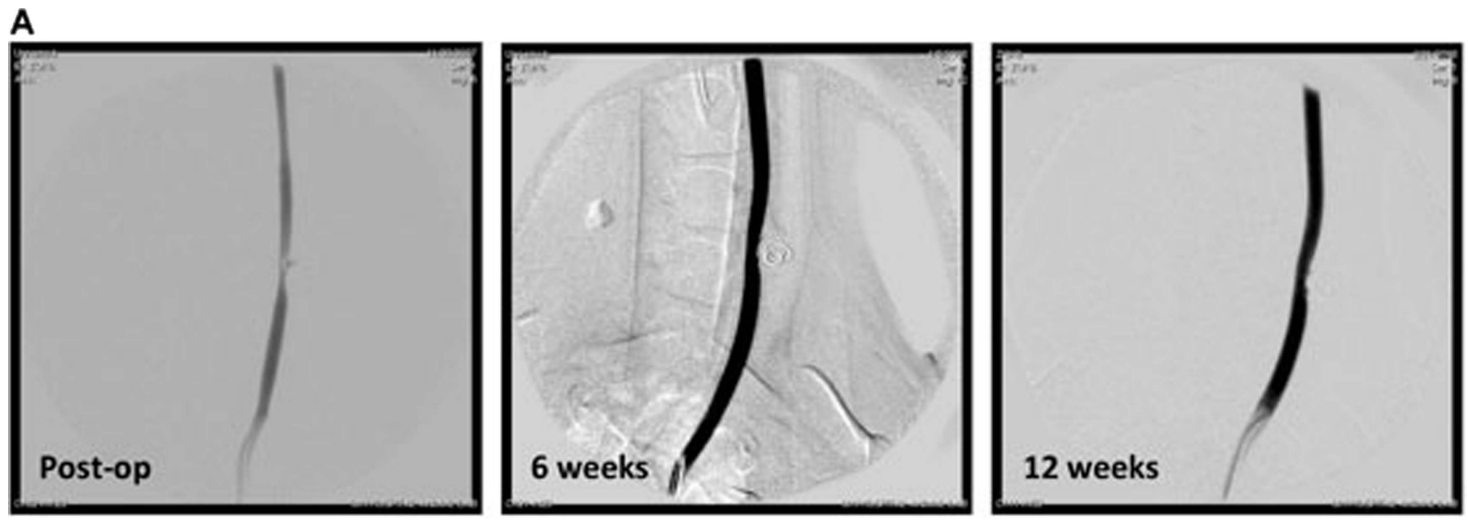

B
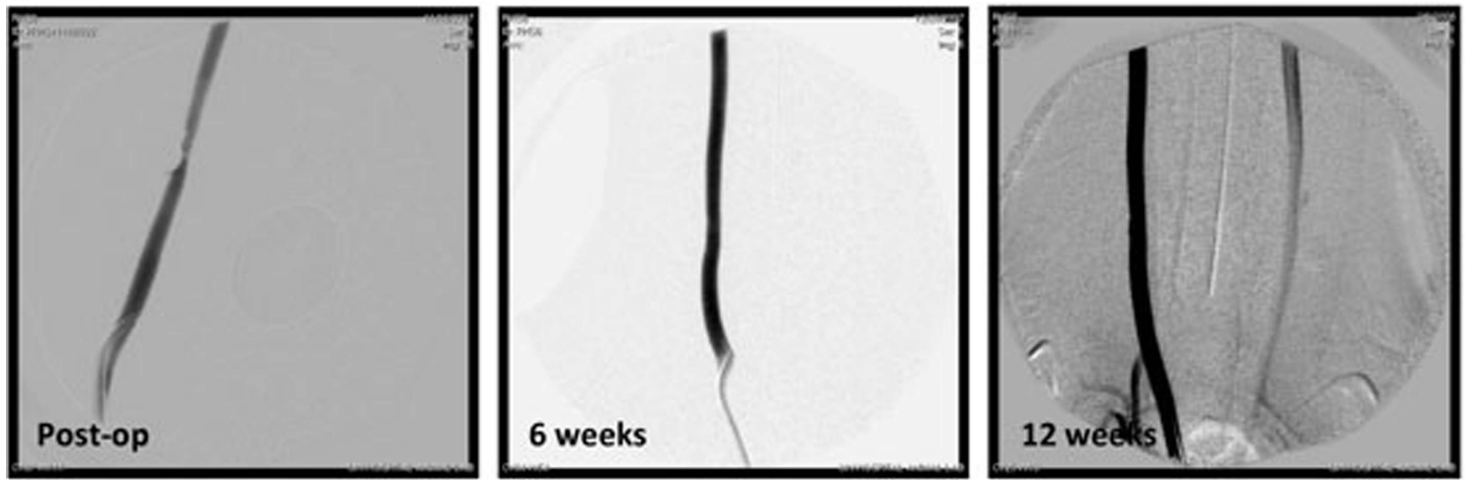

C
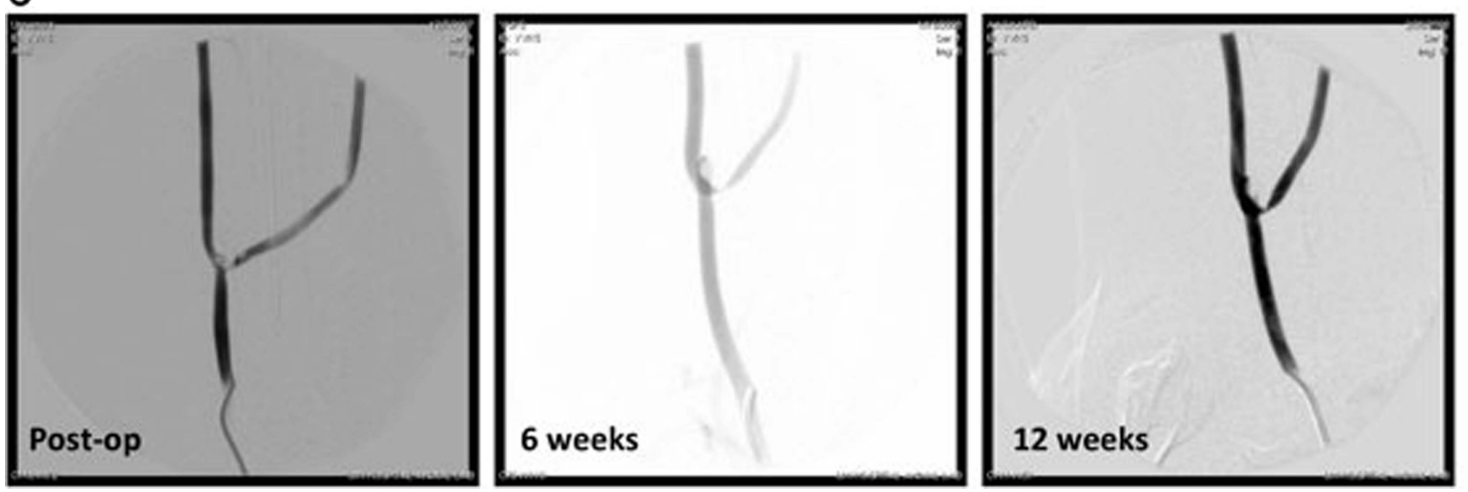

Figure 1 Angiography of (A) sidewall aneurysms in canines treated with a coil-in-shell device or (B) a gel-in-shell device. (C) Angiography of a bifurcation aneurysm in a canine treated with a gel-in-shell device.

demonstrating significantly improved occlusion compared with initial device delivery. Conclusions about occlusion and recanalization rates in bifurcation aneurysms could not be made due to the small number of bifurcation aneurysms in this study.

The design of these devices offers many characteristics that may enable improved aneurysm occlusion outcomes. First, the elasticity of the polymer shell not only allows it to fill the aneurysm on insertion of the shape memory $\mathrm{NiTi}$ wire, ${ }^{17}$ but also allows the device to absorb pulsatile pressures and resist compaction. Second, the composition of the shell itself can be tailored to render the polymer an active participant in the healing process. In this case, we used a copolymer of PU-HA, a non-thrombogenic native glycosaminoglycan known to promote cellular functions conducive to wound healing. ${ }^{22}$ We have previously shown that the bioactive properties of the PU-HA material may be tailored by altering the amount and molecular weight of the HA in the copolymer, ${ }^{15}$ and that the polymer retains these properties following exposure to shear stress. ${ }^{16}$ During aneurysm healing, PU-HA is used as a scaffolding to support cell growth and deposition of extracellular matrix at the neck of the aneurysm. Neither bare metal coils nor liquid embolic agents possess intrinsic bioactivity to mediate healing processes. It is important to note that our device relies less on complete initial occlusion of the aneurysm and more on stimulation of tissue coverage at the neck, thereby eliminating the aneurysm from the circulation more effectively over time. Even when initial occlusion was incomplete, the polymer encapsulated devices created enough stability and healing stimulation to enable the formation of a complete neointimal layer (figure 2B and 2D). The average neointimal thickness reported here is similar to the thickness of a normal canine (or human ${ }^{23}$ ) carotid artery, and is substantially thicker than the neointimal coverage reported in other studies that used coils or gels for experimental aneurysm occlusion in canines. ${ }^{24} 25 \mathrm{~A}$ thicker neointimal layer should lessen the probability of 

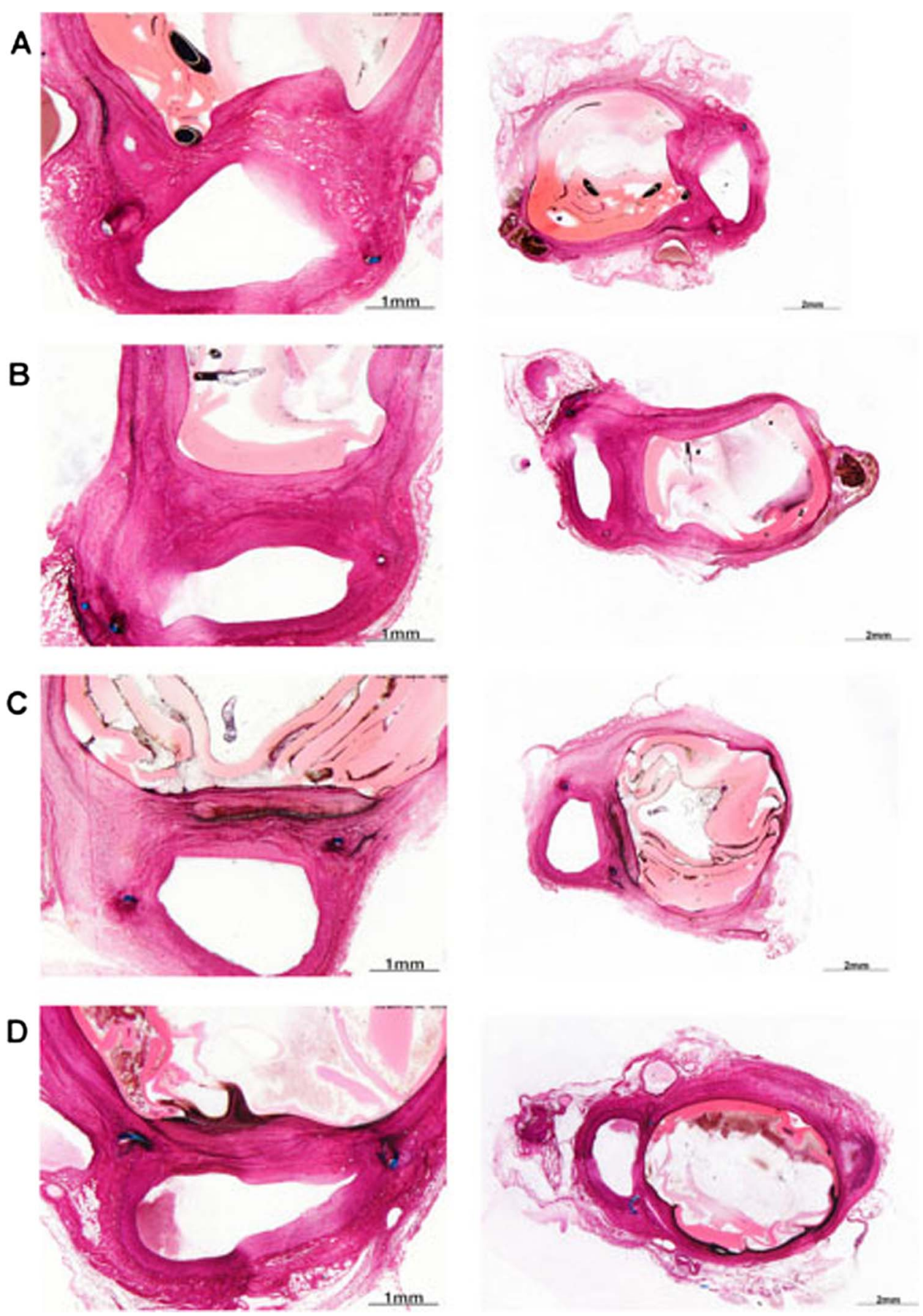

Figure 2 Histological sections of sidewall aneurysms in canines treated with coil-in-shell or gel-in-shell devices, 12 weeks post-implantation. Examples of (A) a coil-in-shell treated aneurysm that was completely occluded at all time points (dog No 2, LCC: $86.3 \%$ occluded at baseline, 93.3\% at week 12), (B) a coil-in-shell treated aneurysm that had incomplete occlusion (dog No 2, RCC: $81.5 \%$ occluded at baseline and at week 12), (C) a gel-in-shell treated aneurysm that was completely occluded at all time points (dog No 3, RCC: $98.4 \%$ occluded at baseline, $100 \%$ at week 12), and (D) a gel-in-shell treated aneurysm that had incomplete occlusion (dog No 5, LCC: $64.7 \%$ occluded at baseline, $67.0 \%$ at week 12).

aneurysmal recurrence and further promote elimination of the aneurysm from the circulation.

Preliminary studies by others have shown that alginate mixed with calcium chloride may be used to successfully occlude aneurysms. ${ }^{26} 27$ We used an alternate chemistry that enabled greater control over gelation kinetics, allowing formation of a homogeneous alginate gel to serve as a conformal space filling material inside the PU-HA shell placed within the aneurysm. The benefits offered by the polymer shell last beyond initial device delivery, as the shell continues to contain the gel, protect against the formation of gel derived emboli during aneurysm healing and, as noted earlier, promotes neointimal coverage at the aneurysm neck. In contrast, neither ethylene vinyl alcohol (the polymer used in current liquid embolics) nor alginate directly support protein adsorption or cell adhesion, ${ }^{28}{ }^{29}$ rendering these materials less effective with respect to promoting the cellular events that are needed to achieve neointimal coverage at the aneurysm neck.

Instances of incomplete occlusion of aneurysms in this study generally occurred due to a need for refinement of the initial 
Table 2 Average neointimal coverage of aneurysms

\begin{tabular}{ll}
\hline Condition & $\begin{array}{l}\text { Neointimal thickness (mm) } \\
\text { (mean (SD)) }\end{array}$ \\
\hline Coil-in-shell, sidewall & $0.662(0.364)$ \\
Gel-in-shell, sidewall & $0.931(0.421)$ \\
Coil-in-shell, bifurcation & $0.362(0.246)$ \\
Gel-in-shell, bifurcation & $0.332(0.203)$ \\
\hline
\end{tabular}

device placement technique, which showed improvement with subsequent implantations. Due to the device delivery approach used for this initial feasibility study, device placement was performed without the assistance of real time angiographic guidance. Additionally, the one instance of device migration was most likely due to a combination of undersizing of the device and improper placement. In this pilot study, the coil-in-shell and gel-in-shell devices were delivered in their fully deployed configurations in order to evaluate the ability of the devices to occlude and heal aneurysms without interference arising from delivery related complications, with plans for future work to address delivery of this device through an endovascular route. The future intended delivery method could consist of placement of a microcatheter in the aneurysm followed by the device being loaded into the microcatheter and subsequent deployment and detachment of the device in the aneurysm. Although delivery related complications were still encountered, the coil-in-shell and gel-in-shell devices proved successful in minimizing aneurysm recurrence and promoting healing, and thus show promise for further investigation for aneurysm occlusion. We did not see significantly better performance of one version of the device over the other. Two versions of the device were developed in order to address drawbacks noted with traditional coils and liquid embolic agents, respectively; this pilot study was intended to test their initial feasibility and performance. Future testing in a larger number of animals and the development of an endovascular delivery method may reveal differential advantages and limitations of these two devices relative to each other. This evaluation is currently underway.

Limitations associated with the AIODs described here include device sizing, which must be precise in order to prevent significant filling of aneurysm. Due to the critical importance of proper device placement in endovascular procedures, an appropriate and accurate endovascular delivery mechanism for our device must also be designed; this is the focus of ongoing work. The cause of the parent artery stenosis observed in some of the cases is also a topic for future investigation.

\section{CONCLUSIONS}

Despite great advances in the development of endovascular techniques to occlude intracranial aneurysms, existing occlusion devices still suffer from numerous drawbacks, and there remains a significant need to develop new strategies that will improve long term aneurysm occlusion and healing. Although much work remains with respect to device deployment, our preliminary work suggests that the design concept presented herein-encapsulation of coils or gel within a bioactive polymer shell-has the potential to facilitate aneurysm occlusion and neointimal healing. The bioactivity of the polymer shell and its ability to form an elastic physical barrier against blood flow proved to be key features that enabled the shell to provide resistance against device compaction and stimulated robust neointimal coverage. Further work will be needed to evaluate AIOD efficacy relative to existing treatments, and whether decreased delivery time and recanalization rates can be achieved with these devices.

Contributors RM, WCC, and KSM conceived the ideas for the work. FX, KH, and CEF constructed the occlusion devices. RM, FX, BA-K, DWC, JRG, KH, CEF, WCC, and KSM performed in vivo implantations and follow-ups. RM, BA-K, and KSM analyzed the data from the in vivo studies. RM, BA-K, WCC, and KSM wrote the manuscript. All authors discussed the results and implications, and commented on the manuscript at all stages.

Funding This work was supported by the WH Coulter Foundation.

Competing interests RM is a consultant for Covidien and Penumbra Inc.

Provenance and peer review Not commissioned; externally peer reviewed.

Data sharing statement Detailed protocols for device construction, and angiographic and histological images for all conditions are available from the corresponding author upon request.

\section{REFERENCES}

1 Cognard C, Weill A, Spelle L, et al. Long-term angiographic follow-up of 169 intracranial berry aneurysms occluded with detachable coils. Radiology 1999;212:348-56.

2 Lee SJ, Cho YD, Kang HS, et al. Coil embolization using the self-expandable closed-cell stent for intracranial saccular aneurysm: a single-center experience of 289 consecutive aneurysms. Clin Radiol 2013;68:256-63.

3 Kurre W, Berkefeld J. Materials and techniques for coiling of cerebral aneurysms: how much scientific evidence do we have? Neuroradiology 2008;50:909-27.

4 Cekirge HS, Saatci I, Ozturk MH, et al. Late angiographic and clinical follow-up results of 100 consecutive aneurysms treated with Onyx reconstruction: largest single-center experience. Neuroradiology 2006;48:113-26.

5 Molyneux AJ, Cekirge S, Saatci I, et al. Cerebral Aneurysm Multicenter European Onyx (CAMEO) trial: results of a prospective observational study in 20 European centers. AJNR Am J Neuroradiol 2004;25:39-51.

6 Norbash AM, Singer RJ. Videographic assessment of the embolic characteristics of three polymeric compounds: ethylene vinyl alcohol, cellulose acetate, and liquid urethane. AJNR Am J Neuroradiol 2001;22:334-40.

7 Struffert T, Roth C, Romeike B, et al. Onyx in an experimental aneurysm model: histological and angiographic results. J Neurosurg 2008;109:77-82.

8 Murayama Y, Vinuela F, Tateshima S, et al. Endovascular treatment of experimental aneurysms by use of a combination of liquid embolic agents and protective devices. AJNR Am J Neuroradiol 2000;21:1726-35.

9 Raymond J, Salazkin I, Metcalfe A, et al. High-concentration ethylene-vinyl alcohol copolymer and endovascular treatment of experimental aneurysms: feasibility of embolization without protection devices at the neck. AJNR Am J Neuroradiol 2003;24:1778-84.

10 Klisch J, Sychra V, Strasilla C, et al. The Woven EndoBridge cerebral aneurysm embolization device (WEB II): initial clinical experience. Neuroradiology 2011;53:599-607.

11 Pierot L, Liebig T, Sychra V, et al. Intrasaccular flow-disruption treatment of intracranial aneurysms: preliminary results of a multicenter clinical study. AJNR Am J Neuroradiol 2012;33:1232-8.

12 Kwon SC, Ding YH, Dai D, et al. Preliminary results of the luna aneurysm embolization system in a rabbit model: a new intrasaccular aneurysm occlusion device. AJNR Am J Neuroradiol 2011;32:602-6.

13 . Piotin $M$, Sourour $N$, Biondi $A$, et al. The LUNA aneurysm embolization system for treatment of intracranial aneurysms. J Neurointervent Surg 2012;4:A16.

14 Xu FM, Hart K, Flanagan CE, et al. A hybrid coil/polymer device for occlusion of cerebral aneurysms. J Med Devices 2009;3.

15 Ruiz A, Flanagan CE, Masters KS. Differential support of cell adhesion and growth by copolymers of polyurethane with hyaluronic acid. J Biomed Mater Res A 2013;101:2870-82.

16 Ruiz A, Rathnam KR, Masters KS. Effect of hyaluronic acid incorporation method on the stability and biological properties of polyurethane-hyaluronic acid biomaterials. J Mater Sci Mater Med 2014;25:487-98.

17 Xu F, Nacker JC, Crone WC, et al. The haemocompatibility of polyurethane-hyaluronic acid copolymers. Biomaterials 2008;29:150-60.

18 Kuo CK, Ma PX. Ionically crosslinked alginate hydrogels as scaffolds for tissue engineering: part 1. Structure, gelation rate and mechanical properties. Biomateria/s 2001;22:511-21

19 Murayama Y, Vinuela F, Suzuki Y, et al. Development of the biologically active Guglielmi detachable coil for the treatment of cerebral aneurysms. Part II: an experimental study in a swine aneurysm model. AJNR Am J Neuroradiol 1999;20:1992-9.

20 Turk AS, Aagaard-Kienitz B, Niemann D, et al. Natural history of the canine vein pouch aneurysm model. AJNR Am J Neuroradiol 2007;28:531-2.

21 Roy D, Milot G, Raymond J. Endovascular treatment of unruptured aneurysms. Stroke 2001;32:1998-2004. 
22 Baier Leach J, Schmidt CE. Hyaluronan. In: Wnek GE, Bowlin GL, eds. Encyclopedia of biomaterials and biomedical engineering. New York, NY: Marcel Dekker, Inc, 2004:779-89.

23 Eigenbrodt ML, Sukhija R, Rose KM, et al. Common carotid artery wall thickness and external diameter as predictors of prevalent and incident cardiac events in a large population study. Cardiovasc Ultrasound 2007;5:11.

24 Raymond J, Berthelet F, Desfaits AC, et al. Cyanoacrylate embolization of experimental aneurysms. AJNR Am J Neuroradiol 2002;23:129-38.

25 Yoshino Y, Niimi Y, Song JK, et al. Endovascular treatment of intracranial aneurysms: comparative evaluation in a terminal bifurcation aneurysm model in dogs. J Neurosurg 2004;101:996-1003.
26 Soga $Y$, Preul MC, Furuse $M$, et al. Calcium alginate provides a high degree of embolization in aneurysm models: a specific comparison to coil packing. Neurosurgery 2004;55:1401-9.

27 Becker TA, Preul MC, Bichard WD, et al. Preliminary investigation of calcium alginate gel as a biocompatible material for endovascular aneurysm embolization in vivo. Neurosurgery 2007;60:1119-27.

28 Augst AD, Kong HJ, Mooney DJ. Alginate hydrogels as biomaterials. Macromol Biosci 2006;6:623-33.

29 Oyane A, Uchida M, Ito A. Laminin-apatite composite coating to enhance cell adhesion to ethylene-vinyl alcohol copolymer. J Biomed Mater Res A 2005;72:168-74. 